|| ISSN(online): 2589-8698 || ISSN(print): 2589-868X ||

International Journal of Medical and Biomedical Studies

Available Online at www.ijmbs.info

NLM (National Library of Medicine ID: 101738825)

Index Copernicus Value 2019: 79.34

Original Research Article

Volume 5, Issue 8; August: 2021; Page No. 295-297

\title{
HOARSENESS OF VOICE: ETIOLOGICAL SPECTRUM
}

\section{Dr Jairaj Kumar Vaishnav}

Assistant Professor, Department of ENT RVRS Medical College Bhilwara (Rajasthan)

Article Info: Received 02 July 2021; Accepted 17 August 2021

DOI: https://doi.org/10.32553/ijmbs.v5i8.2139

Corresponding author: Dr Jairaj Kumar Vaishnav

Conflict of interest: No conflict of interest.

\section{Abstract}

Background: To evaluate the etiological factors of hoarness

Methods: The present study has been carried out in 100 cases of hoarseness were included. Patient with laryngeal and hypopharyngeal malignancy \& up to 15 year of age excluded from the study.

Results: In the present series bronchogenic carcinoma is the most common etiological factor causing vocal cord paralysis and second is thyroid surgery and third is tuberculosis of lung

Conclusion: Voice production is a complex mechanism, involving the muscles of pharynx, palate, tongue, nose and lips. A proper protocol is necessary for identifying the factors responsible for vocal fold paralysis which would help in managing the condition more effectively.

Keywords: Vocal cord, Carcinoma, Paralysis.

\section{Introduction}

Hoarseness are an early and very important symptom of laryngeal pathology caused by simple common cold to the dreaded laryngeal malignancy. ${ }^{1}$ Voice disorders also have a significant influence on vocational, social and emotional adjustments of patients. Mostly vocal cord paralysis is considered as a sign of an underlying pathology, which may be central or peripheral in origin.

Studies have shown peripheral causes (90\%) being more common than central $(10 \%) .^{2}$ Vocal cord paralysis is reported commonly following surgical procedures like thyroidectomy, sometimes as a complication of intubation leading to arytenoid dislocation and rarely reported as an isolated neurologic disease.

The cause for vocal cord paralysis usually arises in the cranial cavity, mediastinum, or neck along the course of corresponding recurrent or inferior laryngeal branch of Vagus. Due to the longer intrathoracic course of the left recurrent laryngeal nerve of left vocal cord is more common than the right. ${ }^{3}$

\section{Material and Methods}

The present study has been carried out in 100 cases of hoarseness were included. Patient with laryngeal and hypopharyngeal malignancy \& up to 15 year of age excluded from the study.

Age and sex of the patient was carefully recorded. Present and past histories taken. Thorough clinical examination was performed and relevant investigation were carried out to come to a definite etiological diagnosis.

Examination of the oral cavity and oropharynx has been done and focal sepsis if any noted. Indirect laryngoscopy was done and finding regarding the type of paralysis, side of paralysis, movement of cords, positions of cord, subglottic and local invasion was recorded.

External examination of neck was done to find out lymphadenopathy, thyroid enlargement, neck injury or scar mark of operation.

Detailed neurological examination was carried out to detect any central or peripheral nerve lesion.

Chest and cardiovascular examination was carried out to exclude any lesion in heart and lung, mediastinum or great vessels.

\section{Observation}

Table 1: Etiological Incidence of vocal cord paralysis

\begin{tabular}{|l|l|l|l|}
\hline S. No. & Etiology & No. of Cases & Percentage \\
\hline 1. & Bronchogenic carcinoma & 23 & $23 \%$ \\
\hline 2. & Thyroid Surgery & 19 & $19 \%$ \\
\hline 3. & Tuberculosis of Lung & 16 & $16 \%$ \\
\hline 4. & Idiopathic & 12 & $12 \%$ \\
\hline 5. & Carcinoma Thyroid & 10 & $10 \%$ \\
\hline
\end{tabular}




\begin{tabular}{|l|l|l|l|}
\hline 6. & Carcinoma oesophagus & 8 & $8 \%$ \\
\hline 7. & Cardiomegaly & 4 & $4 \%$ \\
\hline 8. & Lateral Medullary Syndrome & 4 & $4 \%$ \\
\hline 9. & Neck Injury & 2 & $2 \%$ \\
\hline 10. & Head Injury & 2 & $2 \%$ \\
\hline
\end{tabular}

In the present series bronchogenic carcinoma is the most common etiological factor causing vocal cord paralysis and second is thyroid surgery and third is tuberculosis of lung. Broadly the various etiology factors can be classified according to the site of lesion and to the nature of the lesion the following classification combines these approaches in order to compare this study with those previously reported. The cases in the present series were classified as under:-

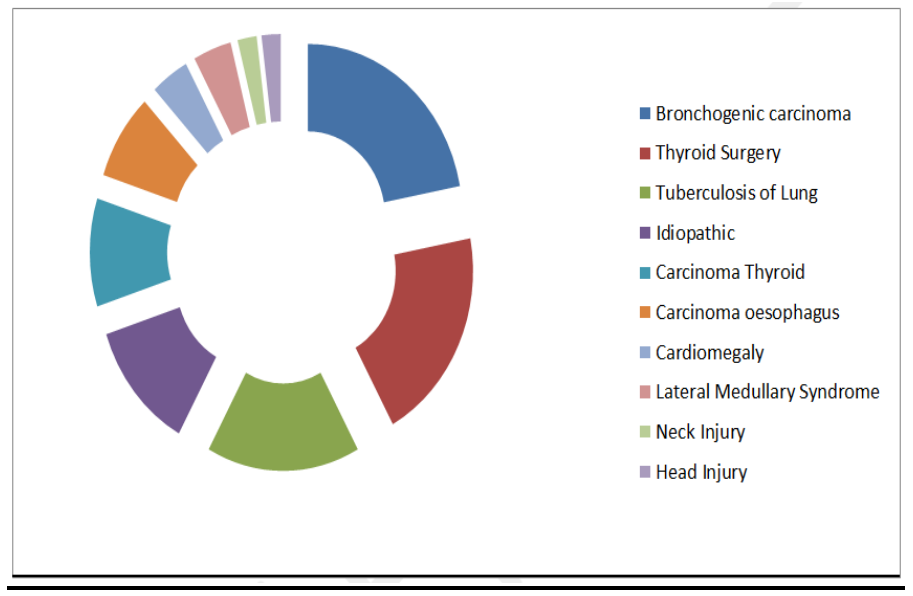

Figure 1:

\section{Discussion}

The vocal cord paralysis is a relatively uncommon condition. The result of our series were compared to six other series. In all series neoplasm and trauma accounted for approximately two third of the total cases. The neoplasm mainly the carcinoma, are most common etiological factor, lung carcinoma being the commonest.

Terries et al (1992) in their study of vocal cord cord paralysis found $40 \%$ cases of neoplasm. ${ }^{4}$ In our study also, 20 cases $(40 \%)$ of vocal cord paralysis were due to neoplasm and out of it 11 cases $(55 \%)$ were due to bronchogenic carcinoma, 5 cases were due to thyroid carcinoma, 4 cases due to carcinoma oesophagus. It was comparable to study of Terris et al (1992).

Bronchogenic carcinoma with mediastinal metastasis is most frequent cause of left recurrent laryngeal nerve paralysis observed in most of the series. This is an indication of unresectability of tumour.

Vocal cord paralysis associated with thyroid tumour indicates the malignant nature of the tumour, it is very rare to suffer paralysis with a benign tumour of the thyroid irrespective of their size, it is therefore, a safe working rule to assume that paralysis of vocal cord occurring in association with a tumour of thyroid gland probably denotes a malignant growth, in our study there were 5 cases of left vocal paralysis due to thyroid malignancy.
Vocal paralysis is usually considered as a poor prognostic sign in malignancy of the lung and oesophagus.

Trauma is the other common cause of vocal cord paralysis in the series of Parnell (47\%), Stenborg(31\%),

Titche $(38 \%))^{5,6}$ In series of yumoto et al and Chen et al $42 \%$ and $48 \%$ cases were only due to trauma. Jayanthy pavithran and Jayakumar menon observed $22 \%$ cases due to trauma. ${ }^{7}$ In all the above series thyroidectomy operation was the most common cause of vocal cord paralysis.

In the present series 11 cases (22\%) were due to trauma in which $10(20 \%)$ cases were due to thyroidectomy, only 1 case $(2 \%)$ due to neck injury.

Mechanism of injury to the RLN during thyroid surgery include complete or partial transaction, traction, or handling of the nerve, contusion, crush, burn, clamping, misplaced ligature, and compromised blood supply.

Mechanical causes of vocal cord paralysis include pressure on RLN by the heart or great vessles and other organomegaly.

Hagan (1963) in his study of 113 cases observed that in $10 \%$ of patients the paralysis was due to mechanical causes (pulmonary fibrosis, tuberculosis). ${ }^{24}$ Parnell (1970) observed in $11 \%$ of his cases, the paralysis was due to pressure on the recurrent nerve. ${ }^{4}$ 
In our study of 50 cases, the vocal cord paralysis due to mechanical factor was observed in 10 cases $(20 \%)$. There were 8 cases of pulmonary tuberculosis and 2 cases of cardiomegaly.

The pulmonary tuberculosis is common in our country due to poor socioeconomic condition as compared to other series.

A lymphnode of the left tracheobronchial group, called the lymph node of the left RLN. It is usually found in relation to the left RLN in the triangle made by aorta, ligamentum arteriosum and the pulmonary artery. In bronchogenic carcinoma and tuberculosis, frequent involvement of nerve secondary to affecting the lymphnode of the left RLN.

\section{Conclusion}

Voice production is a complex mechanism, involving the muscles of pharynx, palate, tongue, nose and lips. A proper protocol is necessary for identifying the factors responsible for vocal fold paralysis which would help in managing the condition more effectively.

\section{References}

1. Samlan RA. Voice analysis. In: Cummings CW, Flint PW, Haughey BH, Robbins KT, et al editors.
Cummings Otorhinolaryngology Head and Neck Surgery.4th ed. Philadelphia: Elsevier Mosby Inc; 2005.p.2008

2. Mc Glashan J. Disorders of the voice. In: Gleeson M, editor. Scott-Brown's Otorhinolaryngology Head and Neck Surgery.7th ed. London: Hodder Arnold; 2008.p.2192-4

3. Vengala RR, Kapilavaya N, Suraneni VR. Evaluation of Clinical Profile and Etiopathology for Hoarseness of Voice- A Study of 146 Cases. Int J Med Res Rev. 2015; 3(2):167-73.

4. Terris DJ, Arnstein DP, Nguyen HH Contemporary evaluation of unilateral vocal cord paralysis. Otolaryngol Head Neck Surg. 1992 Jul;107(1):84-90.

5. Parnell FW, Brandenberg JH. Vocal cord paralysis: A review of 100 cases. Laryngoscope 1970; 80 : 1036-40.

6. Titche L.(1976) Causes of recurrent laryngeal nerve paralysis. Archives of Otolaryngology 102 : 259-61.

7. Jayanthy Pvithran, Jayakumar R Menon: unilateral vocal cord paralysis:An etiopathological study; international journal of phonosurgery and laryngology, jan-jun 2011;1(1);5-10. 